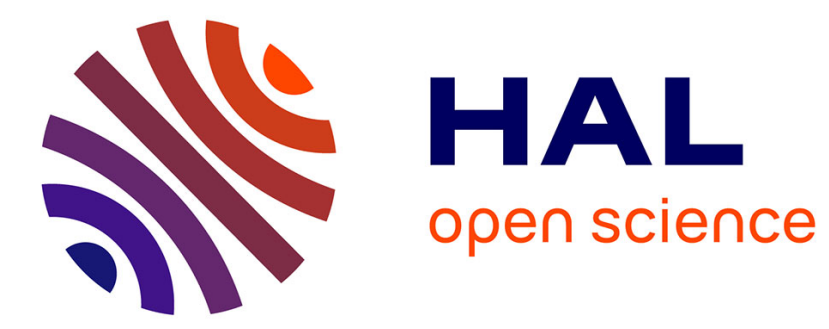

\title{
Quelques réflexions sur la clinique du vieillir
}

François Villa

\section{To cite this version:}

François Villa. Quelques réflexions sur la clinique du vieillir. Psychotherapies, 2013, Psychothérapies, 33 (2013/2), pp.105-113. 10.3917/psys.132.0105 . hal-01494666

\section{HAL Id: hal-01494666 https://hal.science/hal-01494666}

Submitted on 24 Mar 2017

HAL is a multi-disciplinary open access archive for the deposit and dissemination of scientific research documents, whether they are published or not. The documents may come from teaching and research institutions in France or abroad, or from public or private research centers.
L'archive ouverte pluridisciplinaire HAL, est destinée au dépôt et à la diffusion de documents scientifiques de niveau recherche, publiés ou non, émanant des établissements d'enseignement et de recherche français ou étrangers, des laboratoires publics ou privés. 


\title{
QUELQUES RÉFLEXIONS SUR LA CLINIQUE DU VIEILLIR
}

François Villa

\author{
Médecine \& Hygiène | «Psychothérapies »
}

2013/2 Vol. 33 | pages 105 à 113

ISSN 0251-737X

Article disponible en ligne à l'adresse :

http://www.cairn.info/revue-psychotherapies-2013-2-page-105.htm

\section{Pour citer cet article :}

François Villa, "Quelques réflexions sur la clinique du vieillir », Psychothérapies 2013/2 (Vol. 33), p. 105-113.

DOI 10.3917/psys.132.0105

Distribution électronique Cairn.info pour Médecine \& Hygiène.

(C) Médecine \& Hygiène. Tous droits réservés pour tous pays.

La reproduction ou représentation de cet article, notamment par photocopie, n'est autorisée que dans les limites des conditions générales d'utilisation du site ou, le cas échéant, des conditions générales de la licence souscrite par votre établissement. Toute autre reproduction ou représentation, en tout ou partie, sous quelque forme et de quelque manière que ce soit, est interdite sauf accord préalable et écrit de l'éditeur, en dehors des cas prévus par la législation en vigueur en France. Il est précisé que son stockage dans une base de données est également interdit. 


\title{
Quelques réflexions sur la clinique du vieillir
}

\author{
François Villa ${ }^{1}$
}

\section{Résumé}

Cet article est l'occasion pour l'auteur de ressaisir son chemin depuis près de trente ans face aux problématiques psychopathologiques du vieillir. Il lui permet de rappeler les déterminants théoriques de ce qu'il désigne comme la puissance du vieillir. Chacun des temps de cette élaboration a pour origine une expérience clinique concrète ou un moment de vie qui exigeaient, pour pouvoir être pleinement vécus, d'être élaborés. A chaque fois, au-delà de l'échéance factuelle, quelque chose arrivait qui contraignait à approfondir ou à revisiter les concepts, les croyances théoriques. Cette réflexion s'inscrit dans la tradition de la psychopathologie de la vie quotidienne en procédant à un certain nombre de renversements de perspective à partir de la prise en considération que la mort n'est pas la conséquence du vieillissement, mais la mise en échec du processus créatif du vieillir.

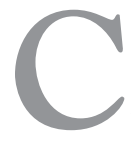
et article sera l'occasion de ressaisir mon cheminement depuis près de trente ans face aux problématiques psychopathologiques du vieillir. Il me permettra de rappeler les déterminants théoriques de ce que je désigne comme la puissance $d u$ vieillir. Chacun de mes temps d'élaboration a toujours eu pour origine une expérience clinique concrète ou un moment de vie qui exigeaient, pour pouvoir être pleinement vécus, d'être élaborés. A chaque fois, audelà de l'échéance factuelle, quelque chose arrivait qui contraignait à approfondir ou à revisiter les concepts, les croyances théoriques. Ma réflexion s'inscrit dans la tradition de la psychopathologie de la vie quotidienne, exprime l'espérance de pouvoir donner un peu de sens à ce qui nous arrive sans que nous ayons pu l'anticiper - c'est-à-dire la plus grande part de notre vie. De nombreux textes, que j'ai repris dans un livre (Villa, 2010), jalonnent un parcours dont la cohérence ne m'est apparue, bien sûr, qu'après-coup.

\section{L'expérience d'une consultation gérontologique}

Ma recherche sur le vieillir s'amorça alors que je commençais à travailler dans une consultation de gérontologie mise en place par une caisse de retraite de cadres. Cette consultation prolongeait la visite annuelle de la médecine du travail que doit passer chaque salarié en France. A titre préventif, la Caisse de prévoyance souhaitait offrir, après la retraite, à chacun de ses cotisants la possibilité d'une telle visite annuelle pour faire le point. Mais cette proposition élargissait le champ de la consultation proposée aux salariés. A côté de la consultation médicale de routine, elle proposait un check-up médical complet avec la possibilité pour ses cotisants de rencontrer un(e) assistant(e) social(e), un(e) sociologue, un(e) psychologue qui leur ferait passer une batterie de tests psychologiques (dont des tests projectifs) et un(e) psychanalyste. A l'occasion de cette expérience professionnelle qui dura un peu plus de quatre ans, je rencontrai près de 2000 personnes dites «âgées». soit à l'occasion de la consultation unique prévue initialement dans le protocole, soit à l'occasion d'une ou deux consultations supplémentaires dont la nécessité s'imposa.

\footnotetext{
Professeur de Psychopathologie, directeur adjoint du Centre de Recherches Psychanalyse, Médecine et Société (EAD N 3522), Univ. Paris Diderot, Sorbonne Paris Cité, 75013 Paris. Psychanalyste, membre de l'Association Psychanalytique de France et de l'Association Psychanalytique Internationale.
} 
Ce fut là une expérience fondatrice. Je noterai, au passage, le caractère rare, voire exceptionnel de cette expérience qui se situait au tout début de ma pratique de psychanalyste. Elle me dérouta et participa des processus de désidéalisation qui jalonnent la vie d'un psychanalyste, l'obligent à s'interroger sur les limites de son art et à ne céder ni au fantasme de toute-puissance de la pensée, ni à une folie à deux.

Parmi les personnes rencontrées à cette occasion, certaines ne se saisissaient, selon elles, de la consultation que par curiosité de l'opportunité de rencontrer cet animal rare que représentait à leurs yeux le psychanalyste. Elles n'avaient pas le sentiment d'une quelconque souffrance psychique qui aurait entravé leur vie dans le passé ou qui l'entraverait aujourd'hui, elles n'avaient, à leur connaissance, pas de demande de traitement psychique. Dans certains cas, ce n'était là bien sûr que le discours manifeste, l'expression d'une conscience qui refoulait, excluait, rejetait l'un des termes du conflit psychique qui les habitait. Mais ce qui fut une expérience surprenante, déconcertante pour moi, c'est que, dans certains cas (que j'évaluerai à environ 40\%), la consultation devait se conclure sur le constat troublant que ces personnes ne relevaient pas de notre art et qu'elles n'avaient pas besoin de nos services. Sous peine de tomber dans la psychanalyse sauvage, nous devions renoncer à toute perspective interprétative. Rester psychanalyste imposait à ce moment-là de renoncer à l'être à tout prix et contraignait à ne pas méconnaître les conditions initiales qui permettent l'instauration d'une situation psychanalytique.

Dans nombre de consultations, je dus me rendre à cette hypothèse : au-delà de tout a priori, de tout jugement moral ou de valeur, de toute représentation-but, l'histoire qui m'était racontée, aussi terne, insipide qu'elle puisse, dans certains cas, me paraître, témoignait d'une solution psychique qui convenait au narrateur. Cette dernière lui avait permis de donner à sa vie une expression qui, ne contrevenant pas de manière dramatique ou douloureuse au principe de plaisir, ne le contraignait pas à se confronter au tragique de la condition humaine. Elle le dispensait, faute de souffrance psychique insupportable, d'avoir à aller au-delà $d u$ principe $d u$ plaisir, à la rencontre du démoniaque de la réalité de la vie psychique pulsionnelle. Dans de nombreux cas, je me suis dit que ces interlocuteurs illustraient l'adage: les gens heureux n'ont pas d'histoire. Nous pourrions avancer que ces personnes pouvaient se permettre, comme l'écri- vait Bion, de ne pas se soucier de découvrir ce que tout le monde sait et que personne ne veut vraiment savoir. Elles n'avaient aucune raison pressante de se contraindre à un travail visant à lever les refoulements qu'exige le processus culturel d'individuation. Elles n'avaient aucune raison de se mettre dans une situation qui, les obligeant à s'approcher de l'inconscient - c'est-à-dire de ce que nous ne connaissons pas et que nous ne voulons pas connaître - les perturberait nécessairement. Elles n'avaient donc, manifestement, aucune raison de se rendre dans ce cabinet du psychanalyste où, selon Bion (1974), devraient se trouver deux individus, le patient et le psychanalyste, assez logiquement effrayés.

\section{Une rencontre déterminante : la curiosité pour la vie psychique, l'amour de la vérité}

Dans d'autres cas, beaucoup plus rares, je fus confronté à une situation étrange. Quelqu'un qui ne souffrait pas psychiquement, qui, a priori, n'avait aucune raison pressante de s'adresser à un psychanalyste et n'avait, à proprement parler, aucune raison sensible de vouloir changer sa vie à propos de laquelle il avait peu de regrets et pas de véritable remord, formulait dans cette consultation une demande de traitement psychique dont le moteur était une curiosité pour sa propre vie psychique et les déterminants inconscients de celle-ci.

Je me souviens ainsi d'une femme de 84 ans qui avait, comme on dit, réussi sa vie. Chercheuse en biologie, elle avait été l'une des premières femmes diplômées dans sa discipline, elle avait travaillé dans l'un des laboratoires les plus prestigieux à l'échelle nationale et internationale de son domaine. Elle avait excellé dans son domaine et avait reçu toutes les reconnaissances imaginables. Son statut de femme «libérée», autonome, indépendante, sa force de caractère ne l'avaient pas empêchée d'avoir une vie amoureuse et sexuelle dont elle était fière, mais lui avait rendu difficile de trouver parmi ses contemporains un partenaire qui accepterait de partager sa vie. Construire un couple ne lui avait été possible que tardivement, vers la quarantaine, avec un homme un peu plus jeune qu'elle; ils s'étaient aimés passionnément et avaient noué une relation d'amants partenaires, confiants l'un dans l'autre. Elle n'avait, 
quant à elle, pas de désir irrépressible d'avoir un enfant, mais son compagnon désirait tellement être père d'un enfant qu'ils feraient ensemble que, malgré son âge, elle avait pris, avec lui, le risque de porter un enfant: une fille était née de cette union. Son mari était mort depuis six ans, elle en avait été profondément triste, il lui manquait encore quotidiennement, mais sans que cela prenne une forme pathologique. Elle entretenait une relation plutôt bonne avec sa fille, surtout depuis que celle-ci avait eu des enfants. Mon interlocutrice avait un petit-fils et une petite-fille qui faisaient son bonheur. Son mari, dont le rêve était d'avoir une famille nombreuse, avait eu la chance de pouvoir les connaître. Cette femme avait toujours eu et avait encore une vie sociale riche. Elle avait des amis qu'elle côtoyait depuis des décennies. Sa réputation de chercheuse lui donnait encore la chance de rencontrer des gens beaucoup plus jeunes qui lui demandaient conseil sur leurs recherches et avec qui elle nouait souvent des liens solides et durables. Passionnée par son domaine, elle ne s'y était pas cantonnée et faisait preuve d'une immense curiosité pour d'autres savoirs et pour les créations culturelles les plus diverses. Elle était fière d'avoir transmis cette passion pour la vie de l'esprit à sa fille et, maintenant, à ses petits-enfants.

Tout allait donc bien et, à l'écouter, aucune perspective de traitement psychique ne se dessinait, mais... mon interlocutrice s'interrogeait. Elle ne regrettait pas ses choix fondamentaux de vie, mais elle ne comprenait pas d'où lui venait la détermination dont elle avait fait montre dès son plus jeune âge. Celle-ci imposait presque toujours le respect aux autres et lui avait permis de réaliser la plupart de ses vœux en tenant bien sûr compte des contraintes sociales, mais... les déterminants de sa vie lui restaient énigmatiques. Comme elle le disait avec humour: "Je n'aimerais pas mourir idiote, si je pouvais comprendre un peu plus ma vie, je n'en mourrais que moins mécontente de devoir partir ». Ce désir l'habitait d'autant plus qu'il lui semblait qu'elle avait sous la main les clés pour comprendre. Elle avait, en effet, deux souvenirs d'enfance dont l'intensité et la permanence la troublaient. Chaque fois qu'ils se présentaient à son esprit, ils la mettaient dans une jubilation et un bonheur qui la surprenaient. Cette expérience excitait d'autant plus sa curiosité que ces souvenirs semblaient anodins et pouvaient paraître relativement insignifiants, mais elle était persuadée qu'ils contenaient les raisons qui avaient déterminé ses choix de vie. A son avis, il suffirait de les déchiffrer en les interprétant pour parvenir à ce gain de compréhension auquel elle aspirait avant de mourir. Mais, me dit-elle, «toute seule je ne parviens pas à les interpréter et je me demandais si une psychanalyse ne me permettrait pas de parvenir à ce résultat, pensez-vous qu'un psychanalyste pourrait m'aider et qu'il accepterait de m'accompagner dans cette aventure? ». Malgré un moment de résistance et d'hésitation de ma part qu'elle eut à surmonter et sans méconnaître l'appétit libidinal de vivre de cette demande ainsi que sa dimension de séduction sexuelle, je finis par accepter de m'engager dans cette aventure. Je n'eus jamais à regretter cette décision, je crois que nous arrivâmes ensemble à entendre les déterminants psychiques qui avaient pesé sur ses choix de vie. Je dois ajouter que je n'ai pas fini encore aujourd'hui de prendre la mesure de ce que je dois à cette rencontre.

Notre travail dura presque dix ans et se termina au matin de sa mort. Nos dernières séances s'étaient tenues chez elle au chevet de son lit qu'elle ne pouvait plus quitter pendant les cinq dernières semaines de sa vie. Le matin de sa mort, elle m'appela et me dit: «Si vous voulez que nous nous rencontrions une dernière fois, il faut que vous veniez ce matin, je sens que je ne serai plus là en fin de journée». Je me rendis, bien évidemment, à ce dernier rendez-vous. Sur cette cure, je n'en dirai pas plus dans cet article, mais, je soulignerai que cette rencontre et quelques autres consultations m'ont appris à être attentif à ce qui, dans le discours ou l'histoire d'un «patient ", constitue un souvenir-écran et peut devenir le pivot par où s'instaure une situation possiblement psychanalytique.

\section{Lâge, une contre-indication?}

Si je me suis attardé sur le point de départ de ma recherche sur les effets du temps sur les processus psychiques et sur le devenir de l'humain, c'est parce que cette expérience fut initiatique et qu'elle porte en elle la plupart des matériaux, des axes et des questions qui m'ont habité et animé depuis près de trois décennies. Cette expérience me conduisit inéluctablement à réfléchir sur la pratique de la psychanalyse, sur ses conditions de possibilité, sur ce qui fait l'opportunité ou non d'une intervention psychanalytique. Mon premier article: «L'âge, une contre- 
indication?», écrit en 1992, est une interrogation sur les conditions de possibilité du travail clinique que j'étais appelé à faire. Je savais que Sigmund Freud avait, à plusieurs reprises et tout au long de son œuvre, avancé que l'âge se révélait être le plus souvent une contre-indication au traitement psychique et, donc, à l'engagement d'une cure psychanalytique. Tenter de saisir les raisons de cette proposition et déterminer ma position par rapport à elle s'avérait une nécessité clinique. De cette clarification ne dépendait rien de moins que ma compréhension de la finalité du travail que je faisais avec les patients reçus dans le cadre de cette consultation.

Dès ce premier article, j'en arrivai assez rapidement à la conclusion que la limite du traitement n'était pas, pour Freud, liée au nombre d'années en lui-même, mais à la dimension économique de la vie psychique. Avec le temps, apparaissent, d'une part, que les conquêtes de la vie de l'esprit ne sauraient réduire à néant ou endiguer définitivement la force du pulsionnel et, d'autre part, que l'accumulation de tout ce qui nous est arrivé, sans être traité psychiquement, accentue l'usure du psychique et en réduit la plasticité créatrice.

Dès ce premier article s'imposa l'idée qu'il était, cliniquement, nécessaire de distinguer vieillir et mourir. Mon expérience clinique me confronta au fait que la mort n'est pas la conséquence du vieillissement, mais l'interruption de ce processus. En forçant le trait, nous avancerons que la mort ne survient que lorsque que nous ne sommes plus capables de vieillir pour continuer à vivre. Vieillir est, dès lors, ce pouvoir qu'a acquis le vivant dans le cours de l'évolution de ne pas mourir trop rapidement, c'est une invention singulière de survie. Comme le dit le père de Daniel Pennac (1997) qu'il cite en exergue de La Fée Carabine: "Vieillir, hélas, mais c’est le seul moyen que j'ai trouvé de ne pas mourir jeune ». Si, plutôt que de parler de vieillissement, je recours au verbe vieillir, c'est pour souligner que nous ne subissons pas passivement le processus du vieillissement. Dans celui-ci existe la dimension subjective d'un acte. Une telle «découverte» changea mon regard clinique et détermina un positionnement technique dans le traitement. Reconnaître la puissance créatrice dont dispose la dynamique $d u$ vieillir nous impose de déconstruire le phénomène pour en dégager le processus dans lequel s'exprime cette puissance. Il s'agit alors de la penser et d'en reconnaître les mécanismes.
De fait, hormis les indéniables injures $d u$ temps, il nous faut reconnaître qu'il n'existe pas aujourd'hui de définition scientifique assurée de la vieillesse, ni du point de vue biologique, ni du point de vue psychologique. Du point de vue des recherches biologiques, existent des hypothèses, dont aucune ne s'est imposée comme unique et globale. C'est ainsi que nous avons été conduit à interroger la pertinence clinique de la notion commune de personne âgée et à montrer qu'il est nécessaire de ne pas se laisser, dans le cours d'un entretien, guider par une telle catégorie. Si un clinicien s'en tenait uniquement à l'âge d'une personne pour déterminer le travail à accomplir, il ne ferait que céder sur sa fonction en se soumettant aux a priori d'un préjugé personnel déterminé, non seulement par sa propre histoire, mais aussi par les représentations du vieillissement qui prédominent socialement. Nous soutenons que l'âge du patient ne détermine aucune spécificité du travail psychique. Nous devons, par contre, examiner les écueils contretransférentiels que le clinicien aura à surmonter pour ne pas faire obstacle à l'élaboration de la personne qui lui a fait confiance (Villa, 2010).

\section{L'hypothèse d'une série étiologique}

Pour penser cette entité protéiforme qu'est le vieillissement, nous faisons appel à l'hypothèse de la série étiologique par laquelle la théorie freudienne rend compte des formes de la vie psychique. Nous envisageons alors les différentes formes du vieillissement comme autant de valeurs que peut prendre la série étiologique. Dans cette série, nous distinguons quatre éléments: les conditions étiologiques, la cause spécifique, la cause concurrente, et la circonstance occasionnante ou cause déclenchante (Freud, 1895b [1894] et 1895f). Cette dernière intervient en dernier dans la série, elle est un facteur temporel et est l'occasion qui s'offre à la cause spécifique qui est, elle, un facteur quantitatif qui peut varier de lui-même ou du fait des circonstances. Ce dernier facteur est l'élément qui n'est absent d'aucun cas de réalisation de l'effet. Il ne peut le déclencher que si les conditions initiales le permettent. Les conditions étiologiques désignent ces facteurs dont l'absence supprimerait non seulement le pouvoir d'action de la cause mais qui, de plus, la feraient disparaître. Les causes concurrentes peuvent être ou ne pas être présentes, elles renforcent la cause spécifique, mais 
elles ne peuvent engendrer à elles seules les effets observés.

Dans cette série, l'âge doit être mis au rang soit des circonstances occasionnantes, soit des causes concurrentes. Pour être le plus rigoureux possible, nous devons tenir compte du fait que certains âges de la vie semblent propices à d'importants remaniements à la fois de la personnalité et des formes de la vie psychique. Dans ces cas, l'âge agit comme cause déclenchante et c'est sa survenue comme dernier élément de la série qui peut le faire hâtivement prendre pour la cause (alors qu'il n'est que la goutte que le vase attendait pour déborder). Ces âges sont déterminés biologiquement, socialement ou familialement.

\section{Vieillir: les destins de l'infantile, la négligence de la dimension économique}

La puissance du vieillir se manifeste comme une capacité de traiter psychiquement les excitations pulsionnelles en transformant la quantité d'excitation en qualité psychique. C'est ainsi que nous rendons la vie supportable, appréciable, désirable et que nous l'investissons pour qu'elle dure le plus longtemps possible. Vieillir comporte une aptitude à différer la satisfaction sans pourtant y renoncer, c'est de là que naissent les projets les plus réalistes comme les plus ambitieux ou les plus mégalomanes. Mettre en latence ce qui, actuellement, se révèle intraitable et se transformer en transformant le monde pour se laisser la chance de pouvoir, à un autre moment, reprendre à nouveaux frais les questions laissées en souffrance font partie des procès qui composent le vieillir. Cela impose de garder une ouverture dans notre perception du monde qui nous permette de saisir ce que les hasards de la vie nous offrent comme des truchements favorisant la reconnaissance et la perlaboration transformatrice de l'infantile qui reste en nous inaccompli.

Le narcissisme de chaque humain est mis à mal dès qu'il doit envisager qu'il néglige toujours trop la force du pulsionnel, son caractère foncièrement inéducable, et qu'il doit admettre que la pulsion constitue une contrainte à un «plus de travail » qui s'impose inexorablement à la vie psychique. Notre idéalisation des fonctions intellectuelles dites supérieures est alors battue en brèche et nous devons envisager qu'avec le temps, les logiques radicales du pulsionnel auront le dernier mot en imposant la disparition du psychique et le retour à l'inanimé et l'anorganique. Lorsqu'on prend la mesure de cette inévitable négligence du moi pour la dimension économique de la vie psychique, on doit penser la matérialité effective de cette vie psychique. S'impose alors la contrainte de penser l'au-delà des représentations du corps et de ses métamorphoses dans le temps. Comme conséquence de cela, nous avons exploré les voies par où s'effectue la reconnaissance de ce que nous appelons la chair du psychique.

Mais avant de poursuivre, arrêtons-nous un instant sur ce que j'appelle la négligence de l'économique. C'est une remarque de Freud ${ }^{2}$ dans «L'analyse avec fin et l'analyse sans fin » qui est à l'origine de ma réflexion. Dans ce paragraphe du texte, Freud relie le sentiment de honte qu'il éprouve à évoquer toutes ces discussions pesantes au fait qu'il aurait «négligé la plupart du temps dans les représentations théoriques de tenir compte du point de vue économique». En premier lieu, il fut plus que déroutant pour moi d'entendre Freud écrire que, lui, l'inventeur de la pulsion et de sa forme la plus radicale, la pulsion de mort, a négligé l'économique. Mais, dans un second temps, il m'apparut nécessaire et opportun d'envisager qu'au travers de cette remarque, nous est indiqué l'un des enjeux cruciaux de notre pratique. Celui-ci détermine la visée même de la cure et contient les conditions de possibilité de sa fin.

L'humain n'est-il pas, en effet, celui qui, au nom des terres du Zuiderzee déjà asséchées, tend à méconnaître toutes celles qui sont encore sous l'eau? N'est-il pas celui qui, toujours, tend à oublier que les digues n'abolissent pas l'eau, tout au plus la contiennent-elles - jamais complètement, jamais définitivement et pas toujours contre vents et marées? L'humain, tout à la fierté des conquêtes de la vie de l'esprit, tout au triomphe de ce qui de la pulsion a pu trouver représentant psychique, n'a-t-il pas toujours trop tendance à vouloir ignorer ce qui de la

2 «J'ai ici l'impression que je devrais avoir honte de toutes ces discussions pesantes, ce qu'elles disent étant connu depuis longtemps et allant de soi. Effectivement nous nous sommes toujours comportés comme si nous savions cela, à cette réserve près, que dans nos représentations théoriques nous avons négligé la plupart du temps de tenir compte du point de vue économique dans la même mesure que des points de vue dynamique et topique. Mon excuse, c'est donc que je remets en mémoire cette négligence» (Freud, 1937c, pp. 241-242). 
pulsion ne parvient pas à être lié psychiquement et reste non pas tant délié qu'inlié? Cela persiste en tant que poussée, morceau d'activité qui agit inlassablement, usant les digues au-delà desquelles la conscience voudrait la rejeter et la maintenir définitivement. Eau travaillant derrière les digues et cheval de Troie (sous la forme des symptômes) dans le Moi qui les introduit comme partie de lui-même, sans se rendre compte qu'il a fait entrer par la grande porte ce qu'il avait cru chasser par la fenêtre.

Nous soutiendrons que la négligence du point de vue économique est déterminée par le fonctionnement même de l'appareil psychique dont elle est un effet second, mais promis à un riche avenir. Émergeant pour résoudre la question de l'excessive pulsion, pour liquider l'indésirable excitation, l'appareil ne s'avère pouvoir y parvenir que partiellement. Sa fonction est vouée à durer en raison même de son échec et elle se modifie par l'adaptation à la situation d'échec du principe d'inertie en tant que principe de constance. Ne pouvant régler son compte définitivement et une fois pour toutes à la dimension économique de la pulsion, il va devoir se contenter de diluer cette dimension dans le fractionnement de la quantité que rend possible la connexion du quantum d'affect à des représentations. Bâti sur le modèle de l'arc réflexe, mais incapable d'être effectivement cet arc qui décocherait la flèche pulsionnelle immédiatement, directement, radicalement, l'appareil psychique renonce à s'occuper directement de la pulsion. Il se voit contraint, ne pouvant ignorer son échec, à ne la traiter qu'au travers de ses représentants que sont les affects (petites quantités) et les représentations. Connectant l'affect à la représentation, ou le déconnectant, l'appareil déplace ces quantités et ces représentations selon les topiques et les dynamiques rendues possibles. En procédant ainsi, l'appareil, au travers des solutions de compromis que sont les formations de la vie psychique, crée des zones qui procurent l'illusion momentanée d'être parvenu à une certaine conciliation. Par ce biais, ce qui d'abord se présenta comme étant intraitable devient, en raison de la liaison psychique, relativement traitable. De même, ce qui fut insupportable: expérience de douleur, état d'angoisse automatique, se fait supportable: oscillation de l'expérience de plaisir à celle de déplaisir, signal d'angoisse qui nous offre la ressource d'une situation de danger avant la situation de détresse. Mais tout cela advient sans que l'exigence de la pulsion cesse, pour autant, d'être ce avec quoi nulle véritable conciliation n'est possible.

Fort de toute cette œuvre qui rend possible sa constitution, puis son développement, l'appareil psychique parvient périodiquement, répétitivement et pour des durées de temps non négligeables, à la constance. Il peut alors fonctionner, pendant les temps de constance (qui s'étendent entre deux poussées de l'excitation restée hors l'appareil), comme s'il avait épuisé la question économique. Il peut ainsi provisoirement négliger ce qui reste encore exclusivement du domaine économique. De là nous vient la capacité éphémère, mais néanmoins appréciable (car source d'un certain repos) de négliger la dimension économique jusqu'au moment où nous la retrouvons. De cette retrouvaille, le sentiment de honte est souvent contemporain : honte de notre absence de moyens propres face aux exigences de la vie pulsionnelle. De ce point de vue, la honte serait un affect tout aussi originaire que l'angoisse (Levi, 1963; Zaltzman, 1998; Barazer, 2003 ; Janin, 2007).

\section{Le somatique : un roc d'origine pour le psychique}

C'est par ces détours, ces méconnaissances et ces déformations que se tracent les chemins par lesquels s'effectue la détermination d'une singularité qui se constitue comme telle par la reconnaissance de notre indispensable inscription dans l'espèce. Nous avons donc interrogé la construction du processus d'individuation qui mène à la singularité, qui est autre chose que l'affirmation autocratique de la petite différence à laquelle narcissiquement nous nous accrochons comme emblème identitaire. Je préciserai que si, dans ma réflexion, je me suis beaucoup intéressé au bord interne de la vie psychique que représente le roc d'origine du somatique, du biologique, je n'ignore pas l'autre bord interne de la vie psychique que constituent la psychologie collective et les produits sociaux du travail de culture.

Pour la psychanalyse, la souffrance menace l'homme de trois côtés, je cite Freud (1930): «En provenance $d u$ corps propre qui, voué à la déchéance et à la dissolution, ne peut même pas se passer de la douleur et de l'angoisse comme signaux d'alarme, en provenance du monde extérieur qui peut faire rage contre nous avec des forces surpuissantes, inexorables et destructrices, 
et finalement à partir des relations avec d'autres hommes ». En prenant la mesure de ces trois sources de souffrance qui, inéluctablement, menacent l'homme, nous pouvons saisir que c'est au lieu même du danger - dans les situations extrêmes - que se révèlent les ressources dont dispose l'homme et qui en font un animal d'une robustesse déconcertante, doté d'une extraordinaire détermination à rester en vie. C'est cette dimension que relève Chalamov (1955) quand il écrit: "Je compris l'essentiel: l'homme n'était pas devenu l'homme parce qu'il était la créature de Dieu, ni parce qu'il avait aux mains ce doigt étonnant qu'est le pouce. Il l'était devenu parce qu'il était physiquement le plus robuste, le plus résistant de tous les animaux et, en second lieu, parce qu'il avait forcé son esprit à servir son corps avec profit ». Faute de place, nous n'accorderons pas à cette précieuse remarque de Chalamov tout le développement qu'elle mériterait. Nous nous contenterons de souligner le renversement de perspective auquel elle nous invite. Dans l'expérience extrême des camps sibériens, il redécouvre l'origine sensorielle du psychisme et sa fonction première, qui est d'avoir à répondre de manière transformatrice et symbolisante aux exigences et urgences de la vie qui s'expriment dans une expérience vécue corporellement.

\section{Psychanalyser, c'est apprendre à mourir}

Assez rapidement s'imposa pour moi la décision de ne pas me positionner comme spécialiste du vieillissement. Dès lors que je reconnaissais que la question de l'âge n'avait pas l'importance qu'on lui prêtait, c'est l'expérience effectivement vécue et pensée de notre indépassable finitude qui devenait l'expérience essentielle. Cette finitude est inhérente à la pulsion, la pulsion est porteuse de finitude parce qu'elle vise sa propre fin (dans tous les sens possibles du mot). Ce n'est, peut-être, que dans la perception lors de l'expérience vécue de ce caractère de la pulsion que nous est donnée (perception endopsychique) une idée de la mort et une prise de conscience de son caractère réel et inéluctable. En semblant m'éloigner de mon point de départ, je retrouve de fait la démarche de Michel de Montaigne (1595), je pourrais avancer en le paraphrasant que «psychanalyser, c'est apprendre à mourir ». Le chemin de ma recherche recoupait celui par lequel l'homme essaye d'apprendre à mourir en étant à même de ne pas s'interdire de jouir pleinement des vanités de la vie. Dans ce cheminement, il peut accorder toute leur importance aux détails de la vie, les plus petits et les plus insignifiants car il sont les lieux où niche la possibilité qu'entre exister et vivre il y ait le moins d'écart possible. Notons qu'il pourrait être intéressant de distinguer psychopathologiquement les maladies $d u$ vivre des maladies de l'exister.

Au fil des années, en réalisant ce travail, qui vise par la théorie à réduire l'écart qui, toujours, subsiste entre la réalité de la condition humaine et notre aptitude à supporter d'avoir à la penser, j'ai pris conscience que si la pensée ne nous permettra pas de surmonter l'écart, elle pourra, par contre, accroître l'acuité de notre perception de la douleur qui accompagne l'exister et le vivre sans les rendre pour autant impossibles.

\section{Enigmatique refoulement organique}

Travaillant sur les processus psychiques qui rendent possible le vieillir, je ne pouvais qu'être confronté à la question des destins de la libido au cours du temps de la vie. Inévitablement, j'en suis venu à interroger la matérialité du corps d'où proviennent les excitations sexuelles qui imposent de nouvelles élaborations psychiques. M'est alors apparu, dans toute son ampleur, l'écart qui existe entre la représentation psychique du corps, construite au fil des ans, et le soma sur lequel en son extrémité s'ouvre le Ça (Freud, 1923b). Ce soma se révèle tragiquement comme ce sol d'origine du psychique que celui-ci s'évertue, dans un retour sur son origine, à essayer de saisir, de représenter en n'y parvenant que partiellement. La construction psychique du corps vise à refouler, pour pouvoir l'ignorer, cet inconnu du soma originaire. Pour penser ce hiatus, l'énigmatique notion de refoulement organique de Freud (Villa, 2010) s'est avérée constituer un outil redoutable pour la réflexion analytique dans le temps même où ma recherche permettait d'en accroître la compréhension. Grâce à cette notion, la notion d'érogénéité de l'ensemble du corps se voit dotée d'une pertinence clinique qui ouvre la voie à une compréhension renouvelée de la notion d'hypocondrie, de la fonction de la douleur, de la construction des zones érogènes, de notre rapport à la maladie et à la 
mort. La reconnaissance de l'existence d'un refoulement organique à l'origine de la vie psychique et de la culture nous oblige à reconnaître que nous ne savons pas ce que le corps peut et cela nous contraint à prêter une attention (la plus libre possible de tout a priori) aux moments et événements de la vie où peut se produire une levée partielle de ce refoulement et où s'offre à nous la chance de (re)connaître davantage ce que le corps peut.

\section{La mort qui sculpte le vivant}

Entre «le seul malheur est celui d'être né » et «je n'ai pas trouvé d'autre moyen pour ne pas mourir que de supporter de vieillir et de le faire activement et pleinement», c'est l'inlassable travail de Pénélope que constitue la vie psychique qui est convoqué. Le but de ce travail est de rendre la vie aussi supportable que possible, travail de vie, mais travail qui, pour nous maintenir en vie vivant, ne saurait éviter d'avoir à supporter la présence de la mort et de son œuvre au cœur du vivant, la mort dont l'œuvre crée l'ossature qui nous permet de vivre debout, la mort qui sculpte le vivant (Ameisen, 2003). Il n'est donc pas surprenant qu'à plusieurs moments, ma recherche n'ait pu avancer qu'en prenant en considération l'importance de l'hypothèse freudienne de la pulsion de mort et sa pertinence clinico-théorique. Le conflit entre la pulsion de mort et la pulsion de vie est le signe même du vivant. Celui-ci ne persiste et ne dure que si l'une et l'autre pulsion triomphent définitivement. Vieillir témoigne de l'aptitude à rester en vie en étant traversé, habité par cette tension originelle, par cette lutte pulsionnelle et de la capacité de la conflictualiser psychiquement sans parvenir cependant à concilier complètement les exigences de l'une et de l'autre. Il n'y a nul espoir de réconciliation ni entre ces deux forces, ni entre les logiques différentes qu'elles engendrent. Il existe néanmoins, par l'élaboration psychique du conflit, la possibilité que ne se produise pas un divorce tel entre l'action de l'une et la poussée de l'autre que surviennent des processus d'étrangement ${ }^{3}$ entre la psyché et le soma, entre l'individu et le

3 Pour comprendre cette notion d'étrangement, il faut revenir sur la signification du mot allemand Entfremdung que Freud utilise à plusieurs reprises dans son œuvre, nous renvoyons à l'article «Étrangement» de la «Terminologie raisonnée» (Bourguignon et al., 1989). monde, entre le Moi et le Ça ou entre le Moi et le Surmoi. Ces processus auraient pour effet de rendre la vie insupportable et l'existence trop improbable et ils participent à ce que l'on désigne comme les pathologies du vieillissement.

$\mathrm{Au}$ fur et à mesure que se développait ma recherche, il m'est apparu à chaque fois plus nettement qu'il était indéniable que l'homme préfère de très loin attribuer la puissance des pulsions à des puissances extérieures à lui, plutôt que d'avoir à reconnaître sa fondamentale réalité pulsionnelle. Mais, même en reconnaissant qu'il nous est, peut-être, impossible de réaliser effectivement complètement ce pas psychique, il n'en demeure pas moins qu'à cet impossible nous sommes, par nécessité, condamnés. Car, bien que le combat soit inégal et l'issue connue, il n'en reste pas moins, comme l'écrit Freud, que s'il y avait un choix à faire, on devrait choisir de périr dans un combat loyal avec le destin. Il nous faut convenir, que cela nous plaise ou non, que nous sommes condamnés, comme l'écrivait Nietzsche, à «prêter l'oreille à ce qui dans les entrailles de l'esprit voudrait rester coi».

\section{Involution? Régression?}

C'est dans ce mouvement que j'ai été conduit à distinguer et à différencier nettement les processus d'involution du processus de la régression. Dans le cours de l'involution s'instaurent des états d'irréversibilité alors que les régressions, que l'involution favorise, comportent des ressources de remaniements qui peuvent permettre l'expression d'un champ de possibles jusque-là inutilisés. L'homme peut ainsi s'accroître des réductions qu'il opère ou subit un peu à la manière dont Giacometti envisageait son travail de sculpteur : élargissement des champs perceptifs de la pensée dans une opération de rétrécissement, de rapetissement et de réduction. Mon expérience clinique m'a conduit à penser que c'est lorsque l'homme n'a pas peur de se défaire moïquement qu'il a des chances d'atteindre réellement ce qu'il est.

Le trajet que j'ai suivi dessine une recherche visant à saisir les conditions qui rendent possible de doter la pulsion d'un destin. Par contre, nous ne nous sommes que très peu intéressés à l'étude du destin fixé que constitue telle ou telle pathologie. Le travail du psychanalyste vise à garder ouvert le champ des possibles psychiques en défaisant les fixations 
libidinales qui entravent la dynamique du processus psychique. En reprenant une formule de Maurice Blanchot, nous avancerons que nous visons, au-delà des réponses psychopathologiques déjà apportées, à redonner à la question existentielle dont elles s'originent la chance de se laisser réentendre d'une manière inouie et inédite. Ce qui a fini par s'imposer, c'est que la dimension inachevée de l'appareil psy- chique, son infantilisme natif font de nous des êtres jamais complètement matures, toujours immatures en certaines de nos parties, toujours en voie de construction ou de reconstruction, ne cessant de nous remanier, de nous modifier, en vieillissant, jusqu'à ce que mort s'ensuive.

(Article reçu à la Rédaction le 6.11.2012)

This article is an opportunity for the author to assess his thirty years of experience with psychopathoOummary logical problematics of aging. It enables him to recall the theoretical determinants of what he designates as the process of aging. Working through each one of these stages has at its origin a concrete clinical experience or a moment in life that requires working through in order to be fully lived. Each time, beyond the actual date set to terminate therapy, something happened that needed deeper investigation or for concepts or theoretical beliefs to be revisited. This reflexion is in keeping with the tradition of everyday psychopathology. Its entails undertaking a certain number of reversals of opinion by considering that death is not a consequence of aging but the failure of the creative processes of aging.

\section{Bibliographie}

Ameisen J.-C. (2003): La sculpture du vivant. Paris, Seuil. Barazer Cl. (2003): Ulysse nu et couvert de boue. Rev. Franç. Psychanal., 67 (5): 1789-1794.

Bion W. R. (1974): Entretiens psychanalytiques. Paris, Gallimard, 1980.

Bourguignon A., Cotet P., Laplanche J., Robert F. (1989) : Traduire Freud. Paris, PUF.

Chalamov V. (1955): La pluie, in: Récits de la Kolyma. Paris, Verdier, 2003.

Freud S. (1895b [1894]) : Du bien-fondé à séparer de la neurasthénie un complexe de symptômes déterminé, en tant que «névrose d'angoisse». OCF-P III: 29-58.

Freud S. (1895f) : Sur la critique de la «névrose d'angoisse». OCFP III: 59-78.
Freud S. (1923b [1922]): Le moi et le ça. OCF-P XVI: 255-302.

Freud S. (1930a [1929]) : Le malaise dans la culture. OCF-P XVIII: 245-333.

Freud S. (1937c): L'analyse avec fin et l'analyse sans fin, in: Résultats, idées, problèmes, II, Paris, PUF, 1985, pp. 231-268. Janin Cl. (2007): La honte, ses figures et ses destins. Paris, PUF. Levi P. (1963): La trêve. Paris, Grasset, 1966.

Montaigne M. E. de (1595) : Essais. Paris, Gallimard, 1950.

Pennac D. (1997): La Fée Carabine. Paris, Gallimard.

Villa F. (1992): L'âge, une contre-indication? in: La puissance du vieillir. Paris, PUF, pp. 1-36.

Villa F. (2010): La puissance du vieillir. Paris, PUF.

Zaltzman N. (1998) : De la guérison psychanalytique. Paris, PUF.
Correspondance: Pr François Villa 30, Bd de Strasbourg 75010 Paris villa@univ-paris-diderot.fr 